\title{
Body weight and/or body mass index are related with renal graft function after living donor kidney transplantation?
}

Saraiva A., Silva J., Soares M., Moreira J., Cavaleiro C., Machado H.

Centro Hospitalar do Porto, Dept of Anaesthesiology \& Intensive Care, Porto, Portugal

\section{Background}

Kidney transplantation is the first choice cost effective treatment for end stage renal disease.

Grafts from living donors provide the best chance of long term transplantation success.

Transplantation in obese patients may be associated with higher risks and costs.

The association of obesity and renal graft outcome has been studied, with the investigators retrieving conflicting results.

\section{Goal of the Study}

Evaluate the influence of weight and body mass index (IBM) on the outcome of renal function in living donor kidney transplanted patients.

\section{Methods}

Retrospective observational audit

Living donor KT patients from January 2010 to December 2012.

Data collected from medical records.

BMI calculated by height and weight at time of transplantation and categorized according to World Health Organization

Main outcome: glomerular filtration rate (GFR) at hospital discharge, and 1st, 3rd, 6 th, 12th and 24th month postoperatively.

Results

Statistical analysis : Spearman correlation (significance level $p<0,05$ ).

\begin{tabular}{|l|l|c|}
\hline \multicolumn{3}{|c|}{$\begin{array}{c}\text { Demographic variables } \\
\text { Values presented as \%, or Mean } \pm \text { SD }\end{array}$} \\
\hline \multicolumn{1}{|c|}{ Donors } & Recipients \\
\hline Male (\%) & $23,9 \%$ & $76,1 \%$ \\
\hline Age (years) & $46,8 \pm 10,7$ & $39,0 \pm 14,2$ \\
\hline
\end{tabular}

Recipient medical characterization

Values presented as $\%$, Mean $\pm \mathrm{SD}$; Medium (maximum; minimum)

\begin{tabular}{|l|l|}
\hline ASA physical status III / IV (\%) & 21,$7 ; 78,3$ \\
\hline Hypertension (\%) & 78,3 \\
\hline Dyslipidaemia (\%) & 34,8 \\
\hline Smoking (\%) & 28,3 \\
\hline Diabetes Mellitus (\%) & 8,7 \\
\hline Chronic renal disease duration (months) & $98,59 \pm 77,2$ \\
\hline Weight (Kg) & $67.8(95 ; 42)$ \\
\hline Body Mass Index $\left(\mathrm{Kg} / \mathrm{m}^{2}\right)$ & $23.5(35.7 ; 17)$
\end{tabular}

Procedure variables

Values presented as $\%$, or Mean \pm SD

\begin{tabular}{|l|c|}
\hline Surgery Duration (min.) & $112,3 \pm 58,0$ \\
\hline Intravenous general anaesthesia (\%) & 95,7 \\
\hline Anaesthesia Duration (min.) & $159,2 \pm 62,2$ \\
\hline Cold Ischemia Time (min.) & $219,3 \pm 248,3$ \\
\hline
\end{tabular}

\section{Discussion}

$31 \%$ of the patients were overweight.

There was no significant relation between weight and GFR at any point of follow-up.

A negative relation not statistically significant was noticed with $\mathrm{BMI}$ and GFR at hospital discharge, 1st, 3rd, 6th, 12th and 24th.

High BMI at the time of transplant is associated with increased risk of wound and surgical site infections, delayed graft function, acute rejection, and graft loss. Controlled prospective studies are lacking to validate this association.

\begin{tabular}{|l|c|c|}
\hline \multicolumn{3}{|c|}{ Weight : GFR ( $\mathrm{n}=46)$} \\
\hline \multicolumn{1}{|c|}{ Time } & $\begin{array}{c}\text { Correlation } \\
\text { coefficient }\end{array}$ & P-value \\
\hline Hospital discharge & -0.029 & 0.848 \\
\hline $1^{\text {st }}$ month & -0.027 & 0.861 \\
\hline $3^{\text {th }}$ month & 0.116 & 0.454 \\
\hline $6^{\text {th }}$ month & -0.006 & 0.593 \\
\hline $12^{\text {th }}$ month & 0.58 & 0.716 \\
\hline $24^{\text {th }}$ month & 0.190 & 0.228 \\
\hline
\end{tabular}

\begin{tabular}{l|c|c|}
\multicolumn{3}{|c}{ BMI: GFR $(\mathrm{n}=43)$} \\
\hline \multicolumn{1}{|c|}{ Time } & $\begin{array}{c}\text { Correlation } \\
\text { coefficient }\end{array}$ & P-value \\
\hline Hospital discharge & -0.219 & 0.15 \\
\hline $1^{\text {st }}$ month & -0.15 & 0.33 \\
\hline $3^{\text {th }}$ month & -0.11 & 0.49 \\
\hline $6^{\text {th }}$ month & -0.25 & 0.09 \\
\hline $12^{\text {th }}$ month & $-0,22$ & 0.14 \\
\hline $24^{\text {th }}$ month & -0.12 & 0.43 \\
\hline
\end{tabular}

\section{Conclusion}

Neither weight or BMI were significantly associated with renal graft function, until at least two years postoperatively.

It is urgent to clarify this issue, as many centers are reluctant to transplant overweight patients because of concerns over adverse outcomes.

Transplant Proc. 2014 Novi46(9):29813; Semin Dial. 2013 SepOct;26(5):56877; Am J Nephrol. 2012;36(6):57586.

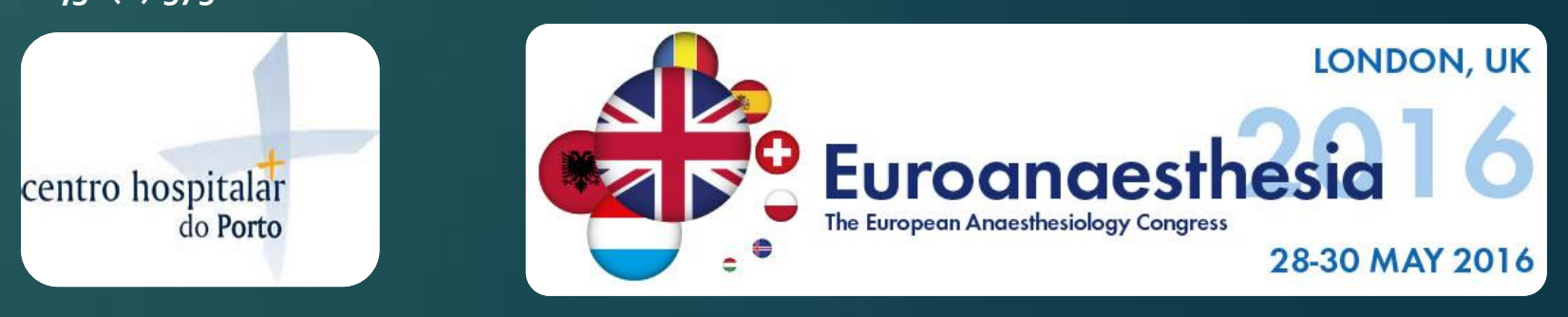

